\title{
Zellen mit Gedächtnis
}

Forscher der Universitätskliniken für Dermatologie und Chirurgie der MedUni Wien konnten unter Leitung von Prof. Dr. Georg Stary einer Untergruppe der zytotoxischen NK-Zellen, die in ihrer Immunantwort bisher als „antigen-unspezifisch“ galten, eine immunologische Erinnerungsfunktion zuschreiben. Rund ein Drittel aller menschlichen NK-Zellen der Leber kann sich demnach an Viren erinnern und somit spezifisch reagieren. Damit gelten diese Zellen als interessantes Target, um sie für das menschliche Immunsystem im Kampf gegen Infektionen und Viren prophylaktisch zu nutzen.

NK-Zellen sind ursprünglich natürliche zytotoxische Killerzellen im menschlichen Blut und gehören zu den Lymphozyten, einer Untergruppe der Leukozyten. Sie sind in der Lage, abnormale Zellen zu erkennen und abzutöten. Den NK-Zellen wurde bisher nachgesagt, dass sie keine Erinnerungsfunktion haben und daher nicht "Antigen-spezifisch“ Zellen abtöten können, sondern nur unspezifisch immer wieder aufs Neue aufViren und Infektionsherde reagieren.

In der in Science Immunology veröffentlichten Studie [1] konnten die Wissenschafter der MedUni Wien jedoch in der Leber - das Organ gilt als Reservoir von NK-Zellen - zeigen, dass es eine Subgruppe von NK-Zellen gibt, die in der Lage ist, etwa Infektionen bei Hepatitis $A$ und $B$ zu bekämpfen und sich auch daran zu erinnern. Diese Untergruppe zeigte auch ein anderes, einzigartiges Gen-Expressionsprofil im Vergleich zu den anderen NK-ZellGruppen.

"Unsere Studienergebnisse zeigen, dass in dieser besonderen Gruppe von NK-Zellen antigenspezifische Prozesse ablaufen und wirksam sind. Daher könnte sich diese NK-Zell-Untergruppe für spezifische, therapeutische, aber auch prophylaktische Impfstrategien eignen", fasst Stary zusammen. Gesunde Menschen haben etwa einen Anteil von 5 bis $15 \%$ an NK-Zellen im Blut, wobei die Leber ein Reservoir für diese Zellen bildet. Als nächsten Schritt untersuchen die Autoren die Rolle dieser NK-Zellen im Verlauf von Infektionskrankheiten. Außerdem wollen sie untersuchen, ob bei Patienten mit seltenen Erkrankungen mit Defekten im Bereich von T- und B-Lymphozyten diese NK-Zellen fehlende Gedächtnisfunktionen zusätzlich übernehmen können.

\section{Literatur \\ 1. Stary V et al (2020) A discrete subset of epi- genetically primed human NK cells mediates antigen-specific immune responses. Sci Immunol. https://doi.org/10.1126/sciimmu-} nol.aba6232

Quelle: Presseaussendung der Medizinischen Universität Wien

Hinweis des Verlags. Der Verlag bleibt in Hinblick auf geografische Zuordnungen und Gebietsbezeichnungen in veröffentlichten Karten und Institutsadressen neutral.

hautnah $2020 \cdot 19: 135$

https://doi.org/10.1007/s12326-02000411-9

(C) Springer-Verlag GmbH Austria, ein Teil von Springer Nature 2020
Hier steht eine Anzeige. Springer 